\title{
Analytische Methoden zum Mineralöl-Wasser-Boden-Komplex
}

\author{
Christian Rübelt \\ Wasserlabor des Instituts für Hygiene und Mikrobiologie der Universität \\ des Saarlandes, Homburg/Saar
}

\begin{abstract}
Analytical methods regarding the mineral oil-water-soil-complex. This paper deals with examples of analyses and methods of identification of oil contaminants in water and soil used in our institute. A method has been developed for the qualitative as well as quantitative estimation of bacterial oil degradation products depending upon bacterial flora, limitation factors and different hydrocarbons or mineral oil products.
\end{abstract}

\section{EINLEITUNG}

Die analytische Bestimmung von Mineralöl wird durch die komplexe Zusammensetzung des Rohöls, dem Ausgangsmaterial der Mineralölprodukte, außerordentlich erschwert. Eine weitere Schwierigkeit liegt in den geringen Konzentrationen, die es in Boden und Wasser zu erfassen gilt, da schon Mengen von $1 \mathrm{mg} / 1$ ( $1 \mathrm{ppm})$ - bei verschiedenen Produkten noch geringere Konzentration - Trinkwasser ungenießbar machen bzw. negative Einflüsse auf Lebewesen im betroffenen Gewässer haben können.

Die folgenden qualitativen und quantitativen analytischen Fragen werden gestellt: (1) Welches Mineralölprodukt ist in der Wasser- oder Bodenprobe enthalten? (2) Welche Olmengen sind in den Proben enthalten? (3) Welche chemischen, physikalischen und mikrobiologischen Veränderungen erfahren die Mineralölprodukte im Wasser und Boden?

Für die Bestimmung von Mineralöl im Wasser muß unterschieden werden zwischen Ol, das als Phase die Wasseroberfläche bedeckt, OI, das sich emulgiert im Wasser befindet, und Olbestandteilen, die echt gelöst im Wasser vorliegen.

Ol auf der Wasseroberfläche kann mit der Methode von Edeline \& BaArs (1967) bestimmt werden und muß auf die Oberfläche bezogen werden. Im Wasser gelöstes oder emulgiertes OI wird in $\mathrm{mg} / \mathrm{l}$ oder ppm angegeben. Der Olgehalt in Bodenproben wird in mg auf $100 \mathrm{~g}$ Boden bezogen.

Ich möchte über die in unserem Institut in diesem Zusammenhang benutzten bzw. entwickelten Methoden berichten, um analytische Möglichkeiten aufzuzeigen. Die umfangreichen Untersuchungen, die zu diesen Methoden geführt haben, bleiben einer gesonderten Veröffentlichung vorbehalten (RüBELT 1968). 


\section{METHODIK UND ERGEBNISSE}

Die Analyse besteht aus den folgenden Schritten: Anreicherung, Extraktion, quantitative Bestimmung im Extrakt und Identifizierung des Ols im Extrakt.

\section{Anreicherung}

Zur Anreicherung benutzen wir Aktivkohlefilter, über die größere Mengen der zu untersuchenden Wasserprobe gepumpt werden. Die auf der Kohle so angereicherten Olspuren werden dann aus der Kohle extrahiert. Eingehende Untersuchungen ergaben, daß mit Aktivkohle nur eine Anreicherung für die Identifizierung der Verunreinigung sinnvoll ist, nicht dagegen für die quantitative OOlbestimmung in der Wasserprobe; dafür ist die Olmenge, die irreversibel auf der Kohle absorbiert wird, zu hoch.
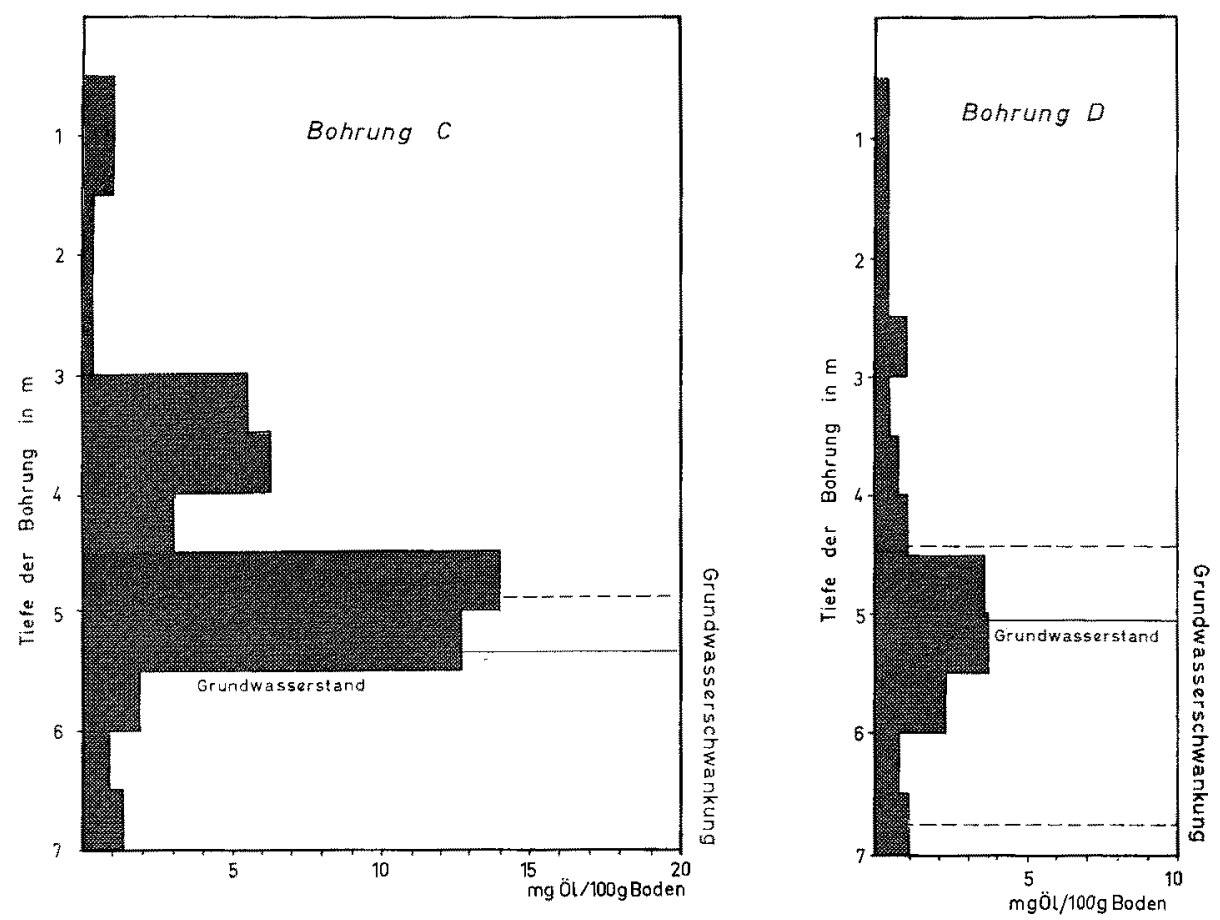

Abb. 1: Charakteristische Bohrkernuntersuchungen (Bohrung $C$ und D)

\section{Extraktion}

Die Wahl des Extraktionsmittels wird durch die Art der anschließenden quantitativen Olbestimmung im Extrakt festgelegt: Soll der Olgehalt nach dem Verdampfen des Extraktionsmittels gravimetrisch bestimmt werden, verwendet man leichtflüchtige Lösungsmittel, um die Verluste der niedrigsiedenden Komponenten des "Ols" niedrig 
$\mathrm{zu}$ halten. Erfolgt die Bestimmung spektralphotometrisch im Infrarot-Bereich zwischen 3,3 und 3,5 $\mu$, so wählt man Tetrachlorkohlenstoff - das einzige Lösungsmittel, das in diesem Bereich keine Eigenabsorption hat (SIMARD et al. 1951).

\section{Quantitative Bestimmungen}

Die IR-Methode ist die schnellste und genaueste „Ol"bestimmungsmethode. Obwohl die Überlegenheit dieser Methode wegen ihrer Anwendbarkeit auf alle Mineralölprodukte und ihrer einfachen Handhabung anderen Verfahren gegenüber durch verschiedene Untersuchungen bestätigt wurde (HEAdington 1953, Ludzack \& WHITFIELD 1956, Lindgren 1957, Rather 1958), blieb der Methode die allgemeine Anwendung wegen der hohen Kosten der IR-Spektralphotometer versagt. Es werden derzeit Versuche unternommen, ein Photometer zu bauen, mit dem Messungen auch im IRBereich durchgeführt werden können und das nur einen Bruchteil der heutigen IRGeräte kosten soll.

Bei Analysen von Was serprobe n (SAwrer \& BeHnke 1959, Bürner 1964, Fastabend 1964, RüBelt et al. 1967) nehmen wir die Proben von 11 in Vierkantflaschen mit 1,5 I Fassungsvermögen, geben im Labor $25 \mathrm{ml} \mathrm{CCl}_{4}$ zu und rühren mit einem hochtourigen Flügelrührer $30 \mathrm{sec}$. Der abgesetzte $\mathrm{CCl}_{4}$-Extrakt wird über wasserfreies $\mathrm{Na}_{2} \mathrm{SO}_{4}$ filtriert und je nach Konzentration in 50-, 10- oder 5-mm-Quarzküvetten im IR-Spektralphotometer zwischen 3,3 und 3,5 $\mu$ gemessen. Die Extinktion ist der Konzentration an "OI“ direkt proportional.

Bei Analysen von $\mathrm{B}$ od e n proben wird von der feuchten Bodenprobe $1 \mathrm{~kg}$ in ein Probenglas eingewogen, mit 15 bis $20 \mathrm{~g}$ wasserfreiem $\mathrm{Na}_{2} \mathrm{SO}_{4}$ versetzt und das Glas mit Gummiring und Klammer verschlossen. $\mathrm{Das} \mathrm{Na}_{2} \mathrm{SO}_{4}$ bindet das im Boden enthaltene Wasser in Form von Kristallwasser. Nach 12 Std. ist der Glasinhalt trokken, ohne daß flüchtige Mineralölanteile verlorengehen. Die Proben werden dann mit $\mathrm{CCl}_{4}$ versetzt. In den meisten Fällen reichen $150 \mathrm{ml}$ für $1 \mathrm{~kg}$ Probe. Durch Umschütteln wird erreicht, daß sich der Tetrachlorkohlenstoff gleichmäßig verteilt. Für die Messung ist es ausreichend, wenn man von dem in der Bodenprobe verteilten $150 \mathrm{ml} \mathrm{CCl}_{4}$ Extrakt $15 \mathrm{ml}$ (für 50-mm-Küvetten) herauspreßt. Die Messung erfolgt wieder im IRSpektralphotometer zwischen 3,3 und 3,6 $\mu$. Aus den Extinktionswerten läßt sich dann die Gesamtmenge „OI" pro kg Bodenprobe errechnen.

Bei Proben, die nicht mineralölspezifische organische Substanzen enthalten, z. B. Abwasser mit pflanzlichen und tierischen Fetten, Boden mit Huminstoffen oder Bodenwachsen, kann man durch Perkolation einer Säule von $5 \mathrm{~g} \mathrm{Al}_{2} \mathrm{O}_{3}$ die Nichtkohlenwasserstoffe abtrennen. Auch hier muß nur eine Küvettenfüllung die Säule passieren.

In unserem Versuchsgelände bei Trier, wo vor 7 Jahren verschiedene Mineralölprodukte im Untergrund infiltriert wurden, konnten wir bei Bohrungen (vg1. Abb. 1, Bohrung $C$ and $D$ ) mit diesem Verfahren die vertikale und horizontale Ausbreitung der Produkte im Boden feststellen.

Durch laufende Wasseranalysen in den Peilrohren können wir die weitere horizontale Ausbreitung des Ols verfolgen. Mit dieser Methode haben wir auch Schlammproben vom Bodensee auf ihren Mineralölgehalt hin untersucht. 


\section{Identifizierung der Olprodukte}

Für die Identifizierung des nachgewiesenen Öls haben wir den folgenden Analysengang entwickelt: Der Extrakt wird strichförmig auf eine Dünnschichtplatte von Kieselgel aufgetragen. Je nach der vorhandenen Menge arbeitet man mit Schichtdicken

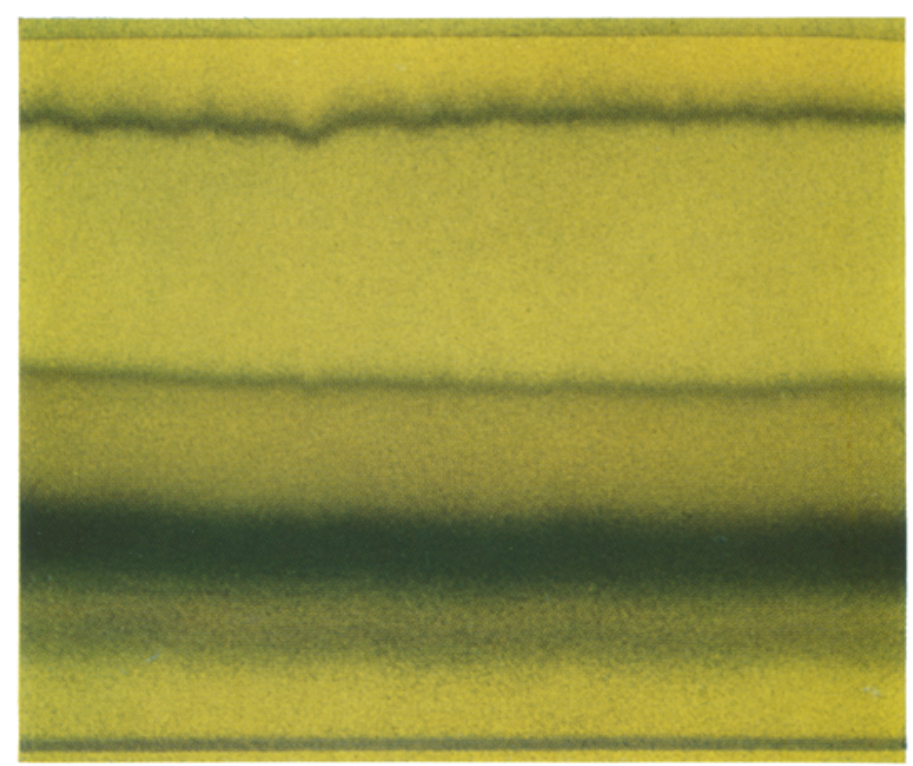

Abb. 2: Auftrennung von $100 \mu 1$ Dieselöl (Rheinpreußen) durch Adsorptions-DC an Kieselgel $\mathrm{G}, 0,5 \mathrm{~mm}$ Schichtdicke, Laufmittel $\mathrm{n}$-Hexan, Laufzeit $35 \mathrm{~min}$, Sprühreagenz 0,03\% Uranin in Wasser

Abb. 3: Auftrennung wie in Abbildung 2 angegeben, unterer Teil mit Sprühreagenz $0,2 \mathrm{ml}$ Formaldehydlösung $(37 \%)$ in $10 \mathrm{ml}$ konz $\mathrm{H}_{2} \mathrm{SO}_{4}$

von 0,3 oder $0,5 \mathrm{~mm}$. Zum strichförmigen Auftragen sollte man unbedingt eines der auf dem Markt befindlichen Auftragegeräte benutzen, da nur bei völlig gleichförmiger Startlinie eine Trennung in einzelne Banden erfolgen kann. Die Platte wird in n-Hexan 
entwickelt. Nachdem das Laufmittel verflüchtigt ist, besprüht man mit einer $0,03 \%$ igen wäßrigen Uraninlösung und kann die Platte im UV-Lichtkasten betrachten (Abb. 2).

Der Extrakt hat sich in verschieden polare Verbindungsklassen getrennt. Aus diesem Bild lassen sich schon Rückschlüsse ziehen, ob es sich um eine Verunreinigung mit einem Teeröl- oder Mineralölprodukt handelt (KRIEGER 1960).

Besprüht man mit Schwefelsäure/Formaldehyd-Reagenz (KucharczyK et al. 1963), so erkennt man die Aromaten als verschiedenfarbige Banden mit jeweils gleicher

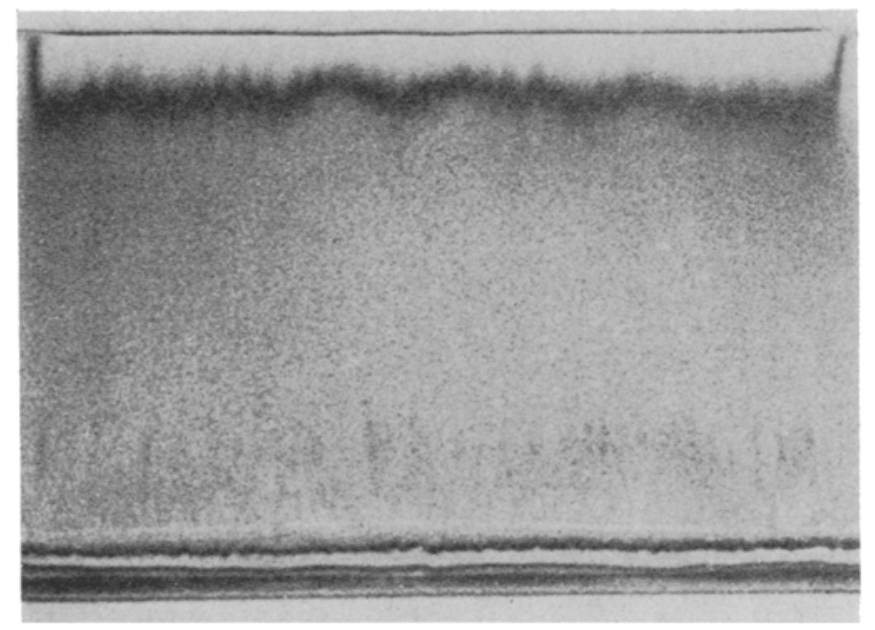

Abb. 4: Auftrennung der Paraffinfraktion aus Dieselöl (Rheinpreußen) in n- und iso-Paraffine auf Harnstoff-imprägnierter Kieselgelplatte. Sprühreagenz $0,03 \%$ Uranin in Wasser

Kernzahl (Abb. 3). Da alle Dieselöle und Heizöle EL im UV das gleiche Bild ergeben, erhält man mit diesem Reagenz eine Differenzierungsmöglichkeit.

Im obersten Band von Abbildung 2 befinden sich die Paraffine. Das Band wird von der Glasplatte abgesaugt, eluiert und die Paraffinlösung auf eine mit Harnstoff imprägnierte Kieselplatte wieder strichförmig aufgetragen. Bei der Entwicklung bilden die n-Paraffine an der Startlinie mit dem Harnstoff Einschlußverbindungen, während die iso-Paraffine mit der Laufmittelfront wandern (Abb. 4). Die n-Paraffine werden eluiert und gaschromatographisch aufgetrennt (Abb. 5). Eine direkte Einspritzung des verunreinigenden Produktes ohne Vortrennung ergibt kein klares Bild (Abb. 6). Aus dem Gaschromatogramm läßt sich der Siedeverlauf der Verunreinigung ermitteln und damit das Mineralölprodukt. Die Analyse dauert 4 Stunden.

Bei dem in den Abbildungen dargestellten Beispiel handelt es sich um einen massiven Oleinbruch in die Kläranlage einer Stadt am Bodensee, der die biologische Stufe und damit die ganze Kläranlage zum Erliegen brachte. Wir erhielten eine schwarzbraune, mit Wasser, Fett und Feststoffen vermischte abgeschöpfte Probe, die stark faulig roch. $100 \mu 1$ dieser Probe wurden für die Analyse benutzt. Die n-Paraffinverteilung erstreckte sich von $\mathrm{C}_{13} \mathrm{H}_{28}$ bis $\mathrm{C}_{37} \mathrm{H}_{76}$, mit dem Maximum bei $\mathrm{C}_{22} \mathrm{H}_{43} / \mathrm{C}_{23} \mathrm{H}_{48}$. Daraus ließ sich der Siedebereich der Probe von $200-500^{\circ} \mathrm{C}$ ableiten. Es muß ein leichtes bis 


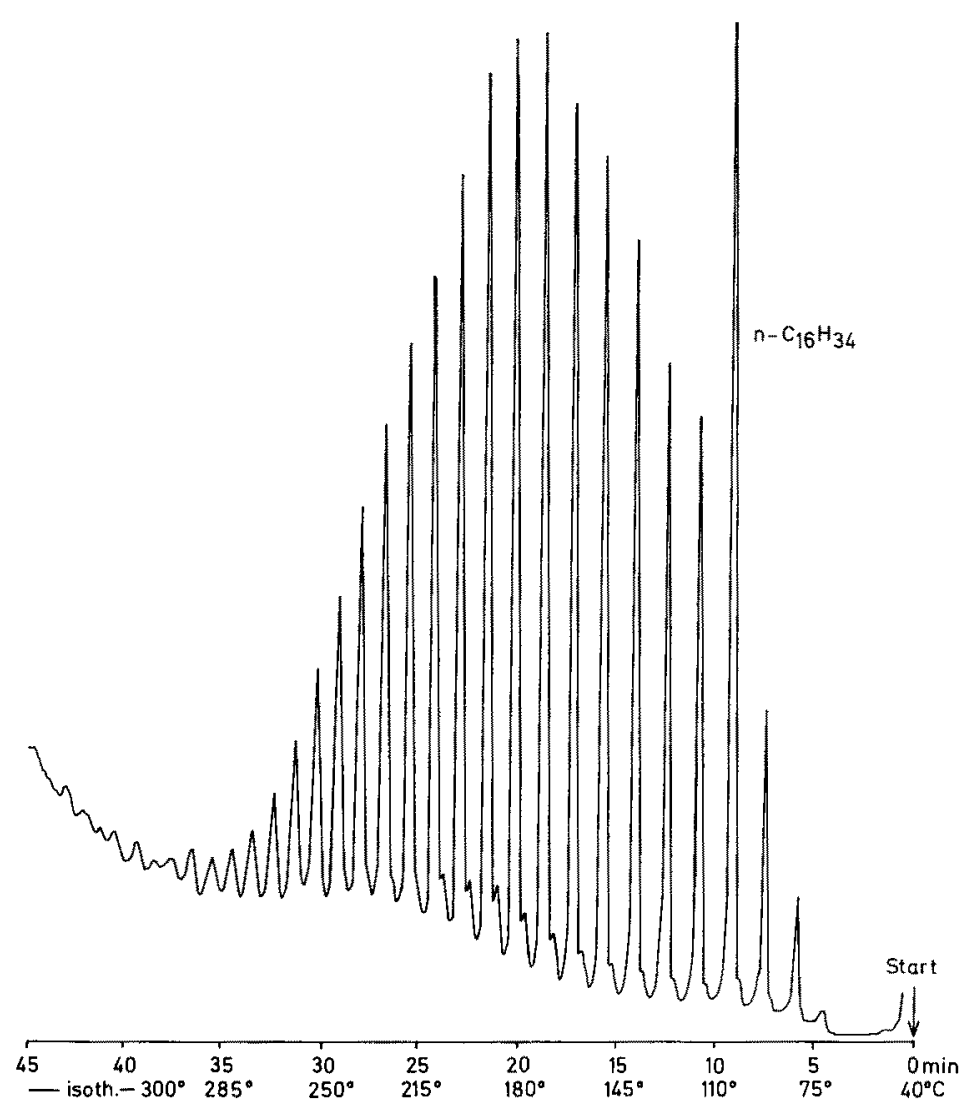

Abb. 5: Gaschromatogramm der n-Paraffinfraktion eines zu identifizierenden Mineralölproduktes. Zur Identifizierung wurde n-Hexadecan zugesetzt. Säule $1 / 8$ " Edelstahl, $120 \mathrm{~cm}, 0,5 \%$

SE-30 auf Chromosorb $60-80$ mesh. Die Banden entsprechen den $\mathrm{C}_{13}-\mathrm{C}_{37}-\mathrm{n}$-Paraffinen

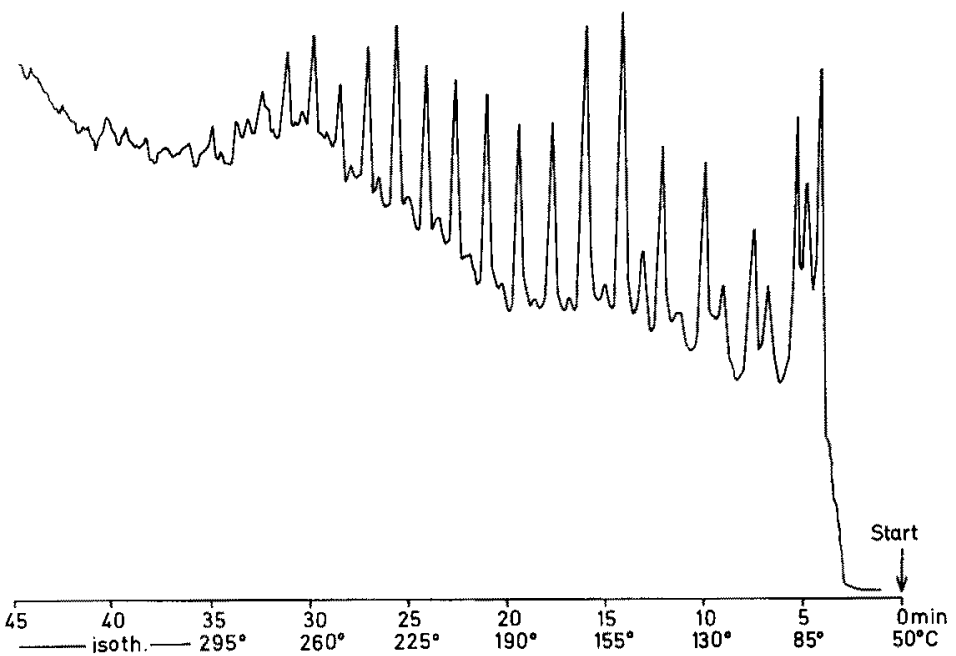

Abb. 6: Gaschromatogramm eines Mineralölproduktes ohne DC-Vortrennung. Gleiche Bedingungen wie in Abbildung 5 angegeben 
mittleres Heizöl gewesen sein, das nur in größeren Anlagen, z. B. zur Kesselheizung, Verwendung findet.

Als unsere Nachweismethoden immer empfindlicher wurden und wir auch in unbeeinflußten Böden und Quellwässern Kohlenwasserstoffe fanden, standen wir vor dem Problem, herauszufinden, wie sich diese natürlich vorkommenden Kohlenwasserstoffe von solchen aus Mineralölprodukten unterscheiden. Die Analytik ist bis jetzt auf die Paraffine ausgerichtet, weil wir uns auf eine Verbindungsklasse beschränken mußten und die Paraffine den Hauptanteil der Mineralölprodukte bilden.
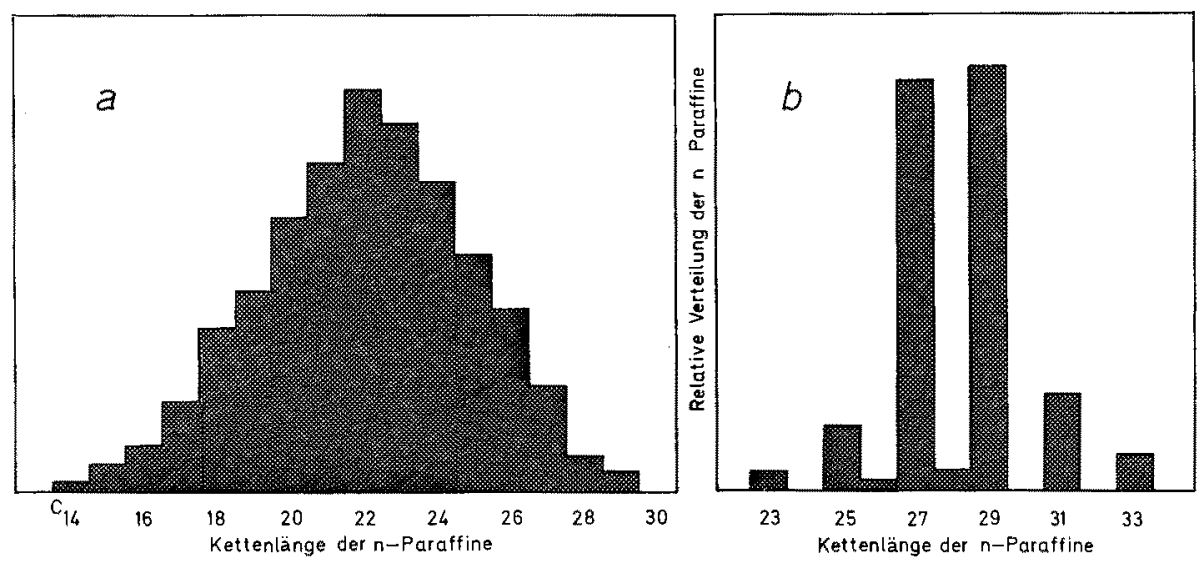

Abb. 7: Verteilung der n-Paraffine (a) in einem Mineralölprodukt, (b) in einer Bodenprobe aus $10 \mathrm{~m}$ Tiefe

Mit dem hier beschriebenen Verfahren ist es nicht nur möglich, Spuren eines Mineralölproduktes zu identifizieren, sondern auch zu entscheiden, ob die gefundenen Kohlenwasserstoffe natürlichen Ursprungs sind oder aus einer Verunreinigung mit einem Mineralölprodukt stammen.

Bei unseren Untersuchungen fanden wir heraus, daß die C-Zahlverteilung bei den n-Paraffinen der natürlichen Paraffine sich von der der Mineralölprodukte unterscheidet: Die natürlich vorkommenden Kohlenwasserstoffe enthalten fast nur ungeradzahlige n-Paraffine, während bei den Mineralölprodukten die Verteilung zwischen geradzahlig und ungeradzahlig 1:1 ist (Abb. 7). Zu analogen Ergebnissen kamen u. a. Stevens 1956, Stevens et al. (1956) und Bray \& Evans (1961) bei der Untersuchung von Meer- und Seesedimenten.

\section{Analytik des mikrobiellen Olabbaues}

Gegenwärtig entwickeln wir eine Analytik, um qualitative und quantitative Aussagen über die beim mikrobiellen Mineralölabbau gebildeten Stoffe machen zu können, und zwar in Abhängigkeit von der Bakterienflora, den limitierenden Faktoren und den verschiedenen Kohlenwasserstoffen bzw. Mineralölprodukten. 
Vom mikrobiellen Abbau von $\mathbf{n}$-Hexadecan in belüfteten Nährsalzlösungen liegen die ersten Ergebnisse vor. Die Bakterienmasse und das Substrat werden extrahiert und der Extrakt auf der Dünnschichtplatte aufgetrennt (Abb. 8). Durch die mitgelaufenen Testsubstanzen lassen sich die einzelnen Verbindungsklassen im Extrakt identifizieren. Diese Flecken oder Banden werden von der Platte abgesaugt, eluiert und gaschromatographisch in die einzelnen Verbindungen aufgetrennt.

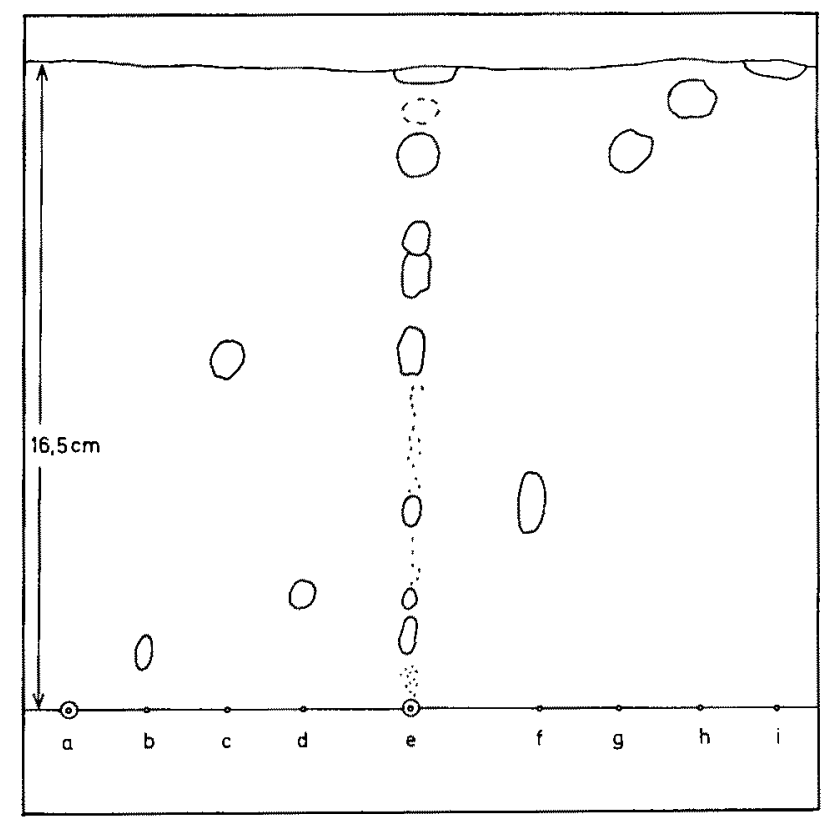

Abb. 8: Trennung der beim mikrobiellen Abbau yon n-Hexadecan gebildeten Stoffe durch Adsoptions-DC an Kieselgel $G$ sauer. Fließmittel n-Hexan-Diäthyläther, $85+15$, Laufzeit 40 min, Sprühreagenz 0,03\% Uranin in Wasser. (a) mono-, (b) di-, (c) tri-Glycerinstearat, (d) Cetylalkohol, (e) Abbauprodukt, (f) Palmitinsäure, (h) Myristinaldehyd, (g) n-Decylstearat, (i) n-Hexadecan

Unsere Ergebnisse bestätigen die Arbeiten von Stewart \& Kallio (1959) und STEWART et al. (1959), daß beim Abbau von n-Hexadecan der Ester der $\mathrm{C}_{16}$-Säure und des $\mathrm{C}_{16}$-Alkohols, das Cetylpalmitat, ein Endprodukt darstellt. Zumindest in diesem einen von uns bisher untersuchten Fall kann man mit Sicherheit sagen, daß das Paraffin nicht vollständig zu $\mathrm{CO}_{2}$ und Wasser abgebaut wird. Veränderungen in der Gasphase sowie die gasförmigen Stoffwechselprodukte werden im Massenspektrometer analysiert.

\section{ZUSAMMENFASSUNG}

1. An Hand von Anwendungsbeispielen aus der Praxis wird über die in unserem Institut angewandten Methoden zum Nachweis und zur Identifizierung von Mineralölverunreinigungen in Wasser- und Bodenproben berichtet. 
2. Für die beim mikrobiellen Mineralölabbau gebildeten Stoffe wird eine Analytik entwickelt, mit der es möglich ist, Cetylpalmitat als ein Endprodukt des n-Hexadecan-Abbaus zu identifizieren.

Mein Dank gilt Herrn Dr. Schweisfurte, der die mikrobiologischen Versuche fachlich betreute, Herrn Dipl-Ing. Penzes, der die Analytik für den mikrobiellen Olabbau entwickelte, und Herm GotTheit für die Anfertigung der Abbildungen.

\section{ZITIERTE LITERATUR}

Bray, E. E. \& Evans, E. D., 1961. Distribution of n-paraffins as a clue to recognition of source beds. Geochim. cosmochim. Acta 22, 2-15.

BürnER, K., 1964. Quantitative Messungen im Bereich der OH-, NH- und CH-Valenzschwingungen. Beckman Report 3, 14-18.

EDELINE, M. F. \& BAARS, J. K., 1967. Water pollution; analysis of hydrocarbons. OECDReport, Paris, 4-5.

FASTABEND, W., 1964. Olverschmutzung in Wasser und Gewässern, Nachweis und quantitative Bestimmung mit dem IR-Spektrophotometer. Dechema-Monogr. 52, 85-98.

Headington, C. E., 1953. Methods for determing hydrocarbons and phenols in water. Analyt. Chem. 25, 1681-1685.

Krieger, H., 1960. Identifizierung von Mineralölen in Böden mit Hilfe der Dünnschichtchromatographie. Gesundheitsingenieur 81, 311-312.

- 1963. Uber ein neues Verfahren zum Nachweis und zur Identifizierung von Mineralölen im Boden auf chromatographischem Wege. Gas- $u$. WassFach 104, 695-698.

Kucharczyk, N., Fohr, J. \& VymĚTAL, J., 1963. Dünnschichtchromatographie von aromatischen Kohlenwasserstoffen und einigen heterocyclischen Verbindungen. J. Chromat. 11, 55-61.

Lindgren, C. G., 1957. Measurement of small quantities of hydrocarbon in water. J. Am. Wat. Wks Ass. 49, 55-63.

Ludzack, F. J. \& Whrrfield, C. E., 1956. Determination of high boiling paraffin hydrocarbons in polluted water. Analyt. Chem. 28, 157-160.

Rather, J. B., 1958. Determination of oil in refinery effluent waters. Analyt. Chem. 30, 36-40.

RüBelt, C., 1966. Spurennachweis von Mineralölprodukten in Bodenproben mit der IRSpektralphotometrie. Analytiker-Tagung vom 13. bis 16. April 1966 in Lindau/Bodensee. $Z$. analyt. Chem. 221, 299-304.

- 1968. Aussagekraft der analytischen Methoden zur Mineralölspurenbestimmung in Bodenund Wasserproben. Saarbrücken, Math.-nat. Diss. 1968.

- Schweisfurth, R. \& ZimmermanN, W., 1967. Experimentaluntersuchungen über die Verschmutzung von Grundwasser durch Mineralölprodukte. T. 2. Gas- u. WassFach 108, $893-900$.

SAWXER, R. R. \& BEHNKE, F.W., 1959. Water pollution analysis using low cost infrared spectrophotometer. The Pittsburgh Conference on analytical chemistry and applied spectroscopy, March 1959. (Vgl. Abwasseranalyse mit Ultrarot-Spektrophotometern niedriger Preislage. 1960. Perkin-Elmer, Überlingen. Tips für die Praxis 5UR.)

Simard, R. G., Hasegawa, J., Bandaruk, W. \& Headington, C. E., 1951. Infrared spectrophotometric determination of oil and phenols in water. Analyt. Chem. 23, 1384-1387.

Stevens, N. P., 1956. Origin of petroleun. Bull. Am. Ass. Petrol. Geol. 40, 51-61.

- Bray, E. E. \& Evans, E. D., 1956. Hydrocarbons in sediments of Gulf of Mexico. Bull. Am. Ass. Petrol. Geol. 40, 975-983.

STEWART, J. E. \& Kaldro, R. E., 1959. Bacterial hydrocarbon oxidation. II. Esters formation from alkanes. J. Bact. 78, 726-730.

- Kallio, R. E., Stevenson D. P., Jones, A. C. \& Schissler, D. O., 1959. Bacterial hydrocarbon oxidation. I. Oxydation of n-hexadecane by a gram-negative coccus. $J$. Bact. 78, 441-448. 